\title{
REVIEW
}

For reprint orders, please contact: reprints@futuremedicine.com

\section{Healthcare-associated infections, infection control and the potential of new antibiotics in development in the USA}

\author{
Alpesh N Amin ${ }^{* 1}$ \& Dennis Deruelle ${ }^{2}$
}

\begin{abstract}
Healthcare-associated infections (HAls) caused by drug-resistant Gramnegative pathogens are a significant burden on the US healthcare system. This problem has been further compounded by the recent decline in the development of new antibiotics targeting Gram-negative organisms. US healthcare agencies have been working to limit the occurrence of HAls by several means, including surveillance systems, prevention practices, antimicrobial stewardship policies and financial incentives. Furthermore, efforts have been made to resume the development of antibiotics in the USA, with the US FDA and US government both implementing acts to boost the number of antibiotics in the clinical pipeline. This review discusses the policies instigated by the US government, including healthcare agencies and the FDA, and describes new antibiotics in development against HAls.
\end{abstract}

\section{Introduction to healthcare-associated infections}

Historically, infections tended to be contracted in the distinct settings of the hospital or community, and were thus defined as hospital- or community-acquired, respectively. More recently, healthcareassociated infections (HAIs) have become an emerging infection type for patients who have had infection detected within $48 \mathrm{~h}$ of hospital admission, and who have also had previous contact with the healthcare service within 1 year [1]. Using data from the National Nosocomial Infections Surveillance (NNIS) system collected between 1990 and 2002, it was estimated that there were 1.7 million HAIs among hospitalized adults and children across the USA in 2002 alone [2]. HAIs caused by Gram-negative pathogens, which are the focus of this review, are a cause of significant healthcare burden in the USA.

\section{- Healthcare-associated infections caused by Gram-negative pathogens}

Of the HAIs reported to the National Healthcare Safety Network (NHSN) in 2009-2010, around $40 \%$ were known to be associated with Gram-negative organisms, including Klebsiella pneumoniae, Acinetobacter baumannii, Pseudomonas aeruginosa and Enterobacter spp. [3]. These Gramnegative organisms, alongside the Gram-positive species Enterococcus faecium and Staphylococcus aureus, have been labeled the ESKAPE pathogens and they cause the majority of HAIs in the USA [4]. Magill et al. found that of the top ten ranked pathogens causing HAIs in their study, four were Gram-negative: Klebsiella spp. (K. pneumoniae/K. oxytoca), Escherichia coli, P. aeruginosa and Enterobacter spp [5].

These Gram-negative pathogens are also associated with drug resistance in the nosocomial setting [6]. In the study by Sievert et al. [3], 20-40\% of healthcare facilities reported at least one

'Department of Medicine, University of California, Irvine, 101 The City Drive South, Orange, CA 92868, USA

${ }^{2}$ IPC Healthcare, 4605 Lankershim BIvd, Ste 617, North Hollywood, CA 91602, USA

*Author for correspondence: Tel.: +1 714456 3785; Fax: +1 714456 7182; anamin@uci.edu

\section{KEYWORDS}

- antibiotic $\bullet$ burden of disease $\bullet$ drug development

- Gram-negative pathogen

- healthcare-associated infection 
Gram-negative isolate, among $K$. pneumoniae, A. baumannii, $P$. aeruginosa and Enterobacter spp., with a resistant phenotype. The lowest frequency of multidrug resistance (MDR) was reported among $E$. coli and Enterobacter spp. $(<5 \%)$, and the highest frequency among $A$. baumannii (44-78\%). The problem of emerging drug resistance among Gram-negative pathogens has been publicized, particularly with the recent decline in antimicrobial research and development [6].

\section{- Current treatment guidelines for healthcare-associated infections}

HAI data collected between 2007 and 2010 showed the following rank order for the same infection types: central line-associated bloodstream infection $(40 \%)$, catheter-associated urinary tract infection (26\%), surgical site infection $(23 \%)$ and ventilator-associated pneumonia (11\%) [3].

The Infectious Diseases Society of America (IDSA) has published guidelines for the management of intravascular catheter-related infections and complicated intra-abdominal infection (cIAI) $[7,8]$. The IDSA in conjunction with American Thoracic Society (ATS) has also produced guidelines for the management of hospital-acquired bacterial pneumonia (HABP) and ventilator-associated bacterial pneumonia (VABP) [9]. No guidelines have been published by the IDSA for complicated urinary tract infection (cUTI). This may be due to the wide variety of patient populations, genitourinary abnormalities and causative pathogens that can be associated with cUTIs [10-12]. The IDSA, however, have published guidelines for the treatment of catheter-associated urinary tract infection, and uncomplicated cystitis and pyelonephritis [8] Additionally, no guidelines have been published by the IDSA for surgical site infections despite surgical site infections being shown to compose up to $23 \%$ of all HAI [13].

This article will review the current preventative and control measures for HAIs being implemented by US healthcare agencies and institutions, including systems for the surveillance of HAIs, antimicrobial stewardship policies to enhance the appropriate use of antibiotics and quality measures to incentivize healthcare facilities to reduce the rate of HAIs. This burden of HAIs has been exacerbated by a recent decline in the development of new antibiotics against Gram-negative pathogens, and this review will also discuss the resumption of antimicrobial research, summarizing the potential, new antimicrobial agents in the pipeline to overcome HAIs caused by Gram-negative organisms.

\section{Controlling healthcare-associated infections}

Many measures, such as surveillance systems, prevention practices, antimicrobial stewardship policies and quality measures, have been implemented to raise the awareness of the healthcare profession to the burden of HAIs and to introduce practices to minimize this burden. This section will describe these measures in further details.

\section{- Surveillance systems}

The NHSN is a program sponsored by the Centers for Disease Control and Prevention (CDC), set up to report and monitor the occurrence of HAIs. The NHSN was formed from the integration of the NNIS system with the National Surveillance System for Health Care Workers and the Dialysis Surveillance Network in 2005 [14]. The CDC also established the Emerging Infections Program in 1995, which is a network of ten US state health departments [15] that runs the Active Bacterial Core surveillance for invasive diseases caused by pathogens such as Haemophilus influenzae, as well as the Healthcare Associated Infections-Community Interface projects for tracking infections and antimicrobial resistance.

The latest national progress report from the CDC, based on data reported to the NHSN, shows that central line-associated bloodstream infections decreased by $44 \%$ (2008-2012), surgical site infections decreased by 20\% (2008-2012) and catheter-associated urinary tract infections increased by 3\% (2009-2012) [16].

\section{- Prevention programs \& practices}

The US Department of Health and Human Services (HHS) comprises 11 operating divisions [17], including the Agency for Healthcare Research and Quality (AHRQ) and the CDC, both of whom have conducted research and written policies aiming to eradicate HAIs from the US healthcare system. The AHRQ supports the Comprehensive Unit-based Safety Program (CUSP), a patient safety model designed to reduce HAIs in a nosocomial environment [18]. In 2003, the AHRQ launched the Keystone ICU project, which used CUSP to lower the rates of 
catheter-related bloodstream infections in over 100 Michigan intensive care units by two-thirds in 3 months [19]. In 2008, the Keystone ICU project teams reported a $50 \%$ improvement in the safety climate of ICUs, attained a median catheter-related blood stream infection rate of zero and reported $99 \%$ compliance with evidencebased ventilator care practices [20]. In 2012, the AHRQ released the CUSP Toolkit, which assists healthcare facilities in understanding how to improve the quality of patient care and to identify potential safety problems. The toolkit is aimed at anyone in the healthcare profession and contains teaching aids, such as slide kits, presentation materials, videos and checklists. Overall, CUSP has reduced catheter-related bloodstream infections by $40 \%$, preventing 2000 infections and saving more than 500 lives [21].

Prevention programs centered on hand washing, decolonization with topical chlorhexidine gluconate, urinary catheter protocols, surgical prophylaxis and screening protocols with eradication have had some success [22]. Hand hygiene has been reported to reduce MRSA infections by $35 \%$ in ICUs [23] and decolonization of all patients by bathing with chlorhexidine gluconate have been shown to prevent HAIs including catheter-related bloodstream infections among ICU patients [24-26].

There has also been some evidence that shortwave ultraviolet radiation might reduce the burden of bacteria [27], which is now being studied in a larger clinical trial [28].

The CDC website lists practical recommendations on how to protect patients from specific HAIs during treatment [29]. In addition, the Association of State and Territorial Health Officials (ASTHO) and CDC published a policy toolkit on the elimination of HAIs [30]. The document provided guidance on the implementation of state policies, such as the provision of financial incentives for HAI prevention, stricter requirements on the certification and training of healthcare providers, enforced adherence to evidence-based practices of infection control, public reporting of data, and ensuring that state HAI legislation is followed.

The HHS has coordinated the efforts of multiple healthcare agencies including several of its own operating divisions and outside organizations, and has set targets to reduce the majority of HAIs by $25-50 \%$ by the year 2020 [31]. The first phase of the action plan focuses on HAIs in acute care hospitals [32]. The document proposes to reduce hospital-acquired HAIs by addressing the following areas: research, information systems and technology, incentives and oversight, and outreach and messaging strategies.

The research aspect of the action plan recommends an increased understanding of the basic science of HAIs, including the key causative pathogens, how they are acquired and the role of biofilm organisms in HAIs. A second recommendation is the strengthening of research focused on the epidemiology of HAIs. One way of achieving this would be to identify the limitations in current surveillance strategies, including labor intensiveness, poor agreement between healthcare professionals when assessing patient cases (inter-rater reliability) and an inability to track all adverse events. These limitations could be addressed by optimizing the use of existing electronic data to measure processes and outcomes. Any gaps in existing epidemiologic knowledge should be identified and explored, for example, identifying emerging MDR organisms and defining the role of vaccines in preventing HAIs.

Additionally, existing infection control prevention measures can be refined and assessed, and additional strategies developed and evaluated through the design and conduct of multicenter trials. Furthermore, a greater number of multicenter projects, like the Keystone project, are needed to elucidate practices that can successfully reduce the rates of HAIs. For practices with strong scientific evidence to support them, research should be conducted on implementing these across institutions.

\section{- Antimicrobial stewardship}

In 2007, the IDSA and the Society for Healthcare Epidemiology of America published guidelines for developing hospital-based programs to enhance antimicrobial stewardship [33]. The guidelines state that the goals of antimicrobial stewardship are to improve clinical outcomes while limiting any negative effects of antibiotic use, such as drug toxicity and pathogen resistance, and to lower healthcare expenditure without compromising the quality of patient care. The document recommendations are outlined in Table 1.

Studies have shown that the successful implementation of an antimicrobial stewardship program can decrease the rates of HAIs [34-36]. Nowak et al. [34] reported a decrease of $9.75 \%$ in antimicrobial expenditures in the first year 
Table 1. Recommendations for the development of an antimicrobial stewardship program.

\begin{tabular}{|c|c|c|}
\hline Subject/area & Actions & Strength of recommendation \\
\hline Antimicrobial stewardship team & $\begin{array}{l}\text { Formation of a multidisciplinary antimicrobial stewardship } \\
\text { team comprising: infectious diseases physician, } \\
\text { clinical pharmacist with infectious diseases training, } \\
\text { hospital administrative support to measure and track }\end{array}$ & $\begin{array}{l}\text { Good evidence from } \geq \text { one well-designed } \\
\text { clinical trial, or based on clinical evidence } \\
\text { descriptive studies or reports of expert } \\
\text { authorities }\end{array}$ \\
\hline
\end{tabular}

\section{antimicrobial use}

Optimal additions: clinical microbiologist and information system specialist to monitor antibiotic resistance, infection control professional and a hospital epidemiologist to advise on improving antimicrobial use

Program implementation

Antimicrobial therapy

Patient care

Other considerations
Development of treatment guidelines based on local microbiology and resistance patterns to improve antibiotic utilization

Prospective audit of antibiotic use through direct interaction and feedback to prescriber, conducted by infectious diseases physician or clinical pharmacist Regulation of antimicrobials included on the hospital formulary and restriction of new agents with limited additional benefit, while monitoring antimicrobial use Education of medical students and hospital staff on prescribing of antimicrobials, with incorporation of prospective audit and intervention

Streamlining of empiric antimicrobial therapy based on microbiological culture results

Adjustment of antibiotic drug dose based on causative pathogen, site of infection, patient characteristics, pharmacokinetic/pharmacodynamics characteristics of drug

Conversion of parenteral antibiotics to oral antibiotics with high bioavailability, when appropriate for the patient

Assisting antimicrobial management of patients through electronic medical records containing data on patientspecific microbiological cultures and susceptibilities, hepatic and renal function, drug-drug interactions Providing patient-specific culture and susceptibility data through clinical microbiology laboratory to enhance antibiotic management of patient and to aid infection control in surveying resistant organisms for epidemiological studies

Antimicrobial order forms to reduce antibiotic usage in longitudinal studies through automatic discontinuation of therapy and requirement of physician justification Antimicrobial cycling (the removal of one antibiotic agent or one class and substitution with an alternative) to prevent or decrease development of drug resistance Combination therapy in certain clinical contexts, such as empiric therapy for critically ill patients with infection caused or suspected to be caused by multidrug resistance pathogens
Good evidence based on clinical evidence descriptive studies, or reports of expert authorities

Good evidence from $\geq$ one randomized, controlled trial

Good evidence from $\geq$ one randomized, controlled trial

Good evidence from $\geq$ one well-designed clinical trial

Good evidence based on clinical evidence, descriptive studies, or reports of expert authorities

Good evidence from $\geq$ one well-designed clinical trial

Good evidence from $\geq$ one well-designed clinical trial

Good evidence based on clinical evidence, descriptive studies, or reports of expert authorities

Good evidence based on clinical evidence, descriptive studies, or reports of expert authorities

Good evidence based on clinical evidence, descriptive studies, or reports of expert authorities

Moderate evidence from $\geq$ one

well-designed clinical trial

Limited evidence from $\geq$ one well-designed clinical trial

Limited evidence from $\geq$ one well-designed clinical trial following implementation of antimicrobial stewardship program, with an estimated overall cumulative cost savings of US\$1.7 million.
Following successful implementation of microbial stewardship program, Prasad et al. [35] reported a reduction in incidence rates of MDR 
K. pneumoniae from $17.7 \%$ in 2011 to $10.5 \%$ in 2013, A. baumanni from 43.3 to $39.5 \%$ and MRSA from 56.8 to $41.6 \%$. Pasquale et al. [36] reported that the interventions made by the antimicrobial stewardship program were associated with significant reductions in the mean length of stay and 30-day all-cause readmission rates for patients with ABSSSI.

Table 2 contains a summary of approaches used for the prevention of HAI [37].

\section{- Quality measures}

Financial penalties have been introduced to further incentivize healthcare institutions to implement the above practice guidelines to minimize HAIs. In 2008, the Centers for Medicare and Medicaid Services (CMS) stopped reimbursing hospitals for the treatment of certain preventable HAIs acquired during hospitalization [38]. This was done in accordance with the Deficit Reduction Act, which was passed by the US Congress in 2005 to decrease federal spending on Medicare and Medicaid services. The act introduced the new Medicare-severity dose-related group (MS-DRG) system, which revised the previous DRG system whereby inpatient prospective payment system (IPPS) hospitals could increase reimbursement for a HAI or hospital-acquired condition. Under the new system, an IPPS hospital cannot increase reimbursement for a hospital-acquired condition that was not present on admission, or for a hospitalacquired condition that is the only comorbidity or complication reported.

In March 2010, the Affordable Care Act was signed into US law, which contained two sections related to improving the quality of hospital care: sections 1886 and 3025 [39]. Section 1886 describes the concept of value-based purchasing, whereby value-based incentive payments are made in a fiscal year to hospitals that meet the required performance standards. Section 3025 describes the reduction of payments to hospitals with high readmission rates.

The Hospital-Acquired Conditions Reduction Program penalizes the lowest quartile hospitals across two domains involving central venous catheter-related infections rates, central lineassociated bloodstream infections and catheter-associated urinary tract infections [40]. These pay-for-performance initiatives apply to Medicare patients but it should be noted that private insurers are adopting this model. This and the other main incentive programs introduced by the CMS, as a result of the Deficit Reduction Act and the Affordable Care Act, are summarized in Table 3.

As well as the implementation of preventative and control measures for HAIs by healthcare agencies and institutions, it is also important that the pharmaceutical industry continue to develop new antibiotics to treat HAIs caused by drug-resistant Gram-negative organisms, after the recent decline in antimicrobial research programs. In the next section, the status of the clinical antibiotic pipeline will be discussed, with a description of the new agents currently in development against Gram-negative pathogens.

\section{Antimicrobial agents for healthcare-associated infections}

Between 1983 and 2012, there was a gradual decrease in the number of US FDA-approved antibiotics, from 16 in 1983-1987 [45] to four from 2009 to the present date (September, 2014) [46]. These four agents, plus ceftobiprole medocaril (currently approved in Europe) are described in Table 4.

Table 2. Summary of approaches used for the prevention of healthcare-associated infections.

\begin{tabular}{|ll|}
\hline Type of approach & Actions \\
Pathogen specific & Active surveillance testing using surveillance systems such as National \\
& Healthcare Safety Network (NHSN) and emerging infections program \\
& Contact precautions \\
& Decolonization \\
Pathogen nonspecific & Antimicrobial stewardship programs such as the Comprehensive \\
& Unit-based Safety Program \\
& Hand washing \\
& Universal decolonization using short-wave ultraviolet radiation or \\
& chlorhexidine gluconate \\
& Universal use of gloves or gloves and gowns \\
Environmental disinfection
\end{tabular}


Table 3. Centres for Medicare and Medicaid Services quality programs for hospitals providing care to patients.

\begin{tabular}{|c|c|c|}
\hline Program & Description & Reward/penalty \\
\hline $\begin{array}{l}\text { Hospital Inpatient Quality Reporting } \\
\text { Program }^{+}\end{array}$ & Hospitals rewarded for reporting designated quality measures & $\begin{array}{l}2 \% \text { reduction to annual update } \\
\text { for hospitals that do not } \\
\text { successfully report }\end{array}$ \\
\hline $\begin{array}{l}\text { Hospital Value-Based Purchasing } \\
\text { Program (IPPS) }\end{array}$ & $\begin{array}{l}\text { Hospitals awarded incentive payments for the quality of care } \\
\text { provided, based on the value-based incentive payment adjustment } \\
\text { factor and the portion of Medicare payments subject to the } \\
\text { adjustment factor }\end{array}$ & $\begin{array}{l}\text { 1.25\% of base operating DRG in } \\
\text { FY } 2014,1.5 \% \text { in FY } 2015\end{array}$ \\
\hline $\begin{array}{l}\text { Hospital Readmissions Reduction } \\
\text { Program }^{\S} \text { (IPPS) }\end{array}$ & $\begin{array}{l}\text { Hospitals penalized for excess readmissions, based on the } \\
\text { readmissions adjustment factor }\end{array}$ & $\begin{array}{l}\text { 2\% reduction in FY 2014, 3\% in } \\
\text { FY } 2015\end{array}$ \\
\hline $\begin{array}{l}\text { Hospital-Acquired Conditions } \\
\text { Reduction Program" }\end{array}$ & $\begin{array}{l}\text { Hospitals in the lowest-performing quartile of hospital-acquired } \\
\text { conditions in domains } 1 \text { and } 2 \text { are not paid at a higher MS-DRG rate } \\
\text { for patients with conditions acquired during hospitalization }\end{array}$ & $1 \%$ payment reduction \\
\hline \multicolumn{3}{|c|}{$\begin{array}{l}\text { 'Data taken from [41]. } \\
\text { "Data taken from [42]. } \\
\text { 'Data taken from [43]. } \\
\text { "Data taken from [40,44]. } \\
\text { CMS: Centres for Medicare and Medicaid Services; DRG: Diagnosis-related group; FY: Fiscal year; IPPS: Inpatient prospective payment system; MS-DRG: Medicare-severity } \\
\text { dose-related group. }\end{array}$} \\
\hline
\end{tabular}

Three of these agents (televancin, dalbavancin and tedizolid) are only active against Gram-positive species. The fourth, ceftaroline fosamil, is active against both Gram-positive and Gram-negative species (excluding A. baumannii, $P$. aeruginosa and Gram-negative strains producing derepressed AmpC or extended-spectrum $\beta$-lactamases [ESBLs]). Unlike earlier cephalosporins, ceftaroline fosamil and ceftobiprole medocaril are active against MRSA, by virtue of their affinity for penicillin-binding protein (PBP) 2a [50].

\section{- Decline of antimicrobial drug development}

The reasons for the overall decrease in new approved antibiotics are both practical and economical. There have been no new antimicrobial classes targeting Gram-negative bacteria in the past 40 years [51] and this may be due, in part, to the more complex structure of Gramnegative compared with Gram-positive bacteria [52]. Furthermore, only a handful of the 20 or so large pharmaceutical companies conducting antimicrobial drug development are still in the field, which may have meant the loss of some expertise [53].

The high costs associated with the development of antibiotics, estimated at US $\$ 1$ billion per drug [54], compared with a relatively low rate of return, mean that there are also economic factors deterring companies from drug development. A monograph from the Office of Health Economics (London, UK) found that the net present value of an antibiotic to a drug company is minus US $\$ 50$ million at discovery [55]. The discovery and development of antimicrobials has also become less attractive to pharmaceutical companies given that these drugs are administered for relatively short courses and they can lose efficacy with overuse as pathogens gain resistance [51]. In addition, antibiotic development is also competing for resources with other therapeutic areas that have higher rates of return for pharmaceutical companies.

\section{- Resumption of antimicrobial research}

To address the problem of drug resistance and reverse the decline in antimicrobial research and development, two important programs have been initiated in the USA. The Generating Antibiotic Incentives Now (GAIN) Act was signed into US law in 2012 as part of the FDA Safety and Innovation Act [56]. The Act states that a drug that is designated a qualified infectious disease product (QIDP) can receive automatic priority review, gain an extra 5-7 years of market exclusivity without competition from generics, and obtain expedited approval from the FDA for being a 'breakthrough therapy.' A list of 'qualifying pathogens,' which are said to have 'the potential to pose a serious threat to public health,' is also given, for example, 'multidrug resistant Gram-negative bacteria, including Acinetobacter, Klebsiella, Pseudomonas and E. coli species' [57].

The $10 \times$ '20 Initiative was launched by the IDSA to 'develop 10 new, safe and effective antibiotics by 2020 ' [58]. These new agents are to 
be systemically administered, and can originate from new or existing antimicrobial classes, but should be targeted to treat MDR infections. Boucher et al. [59] published an update to the IDSA document in 2013, indicating that progress had been made by listing new antibiotics that were in development for the treatment of Gram-negative infections.

\section{- New antibiotics against Gram-negative pathogens}

Table 5 shows the new antibiotics with activity against Gram-negative organisms that are currently in preclinical (pre-Phase II) development. Two of these agents, POL7080 and CTIX1278, belong to novel antibiotic classes, and are synthetic compounds that mimic the action of the immune system cells protegrin (porcine leukocyte peptide) and defensin (human neutrophil peptide), respectively.

Among the antimicrobial agents against Gram-negative organisms that are currently in the later stages of clinical development, there are single-agent antibiotics and those that are a combination of two agents. As a result of the GAIN act, many of these drugs have been designated as a QIDP by the FDA, entitling them to priority review and fast-track status.

Single-agent antibiotics

Eravacycline (TP-434) (Tetraphase Pharmaceuticals, MA, USA) is a fluorocycline tetracycline antibiotic with in vitro activity against $A$. baumannii, $H$. influenzae and Enterobacteriaceae (including ESBL-producing isolates) [68]. In a Phase II study for the treatment of community-acquired

\section{Table 4. New antibiotics approved since 2009.}

\begin{tabular}{|c|c|c|c|c|c|}
\hline Agent name & Company & Antibiotic class & Indication & Pathogens & Date of approval \\
\hline \multirow[t]{2}{*}{$\begin{array}{l}\text { Ceftaroline } \\
\text { fosamil }^{+}\end{array}$} & \multirow[t]{2}{*}{$\begin{array}{l}\text { Forest Laboratories } \\
\text { (NY, USA) }\end{array}$} & \multirow[t]{2}{*}{ Cephalosporin } & ABSSSI & $\begin{array}{l}\text { Staphylococcus aureus (including MSSA } \\
\text { and MRSA), Staphylococcus pyogenes, } \\
\text { Staphylococcus agalactiae, Escherichia coli, } \\
\text { Klebsiella pneumoniae, Klebsiella oxytoca }\end{array}$ & \multirow[t]{2}{*}{ November 2010} \\
\hline & & & CABP & $\begin{array}{l}\text { Streptococcus pneumoniae, S. aureus } \\
\text { (MSSA only), Haemophilus influenzae, } \\
\text { K. pneumoniae, K. oxytoca, E. coli }\end{array}$ & \\
\hline Dalbavancin & $\begin{array}{l}\text { Durata } \\
\text { Therapeutics } \\
\text { (IL, USA) }\end{array}$ & Lipoglycopeptide & ABSSSI & $\begin{array}{l}\text { S. aureus (including MSSA and MRSA), } \\
\text { S.pyogenes, S. agalactiae and Streptococcus } \\
\text { anginosus group (including Streptococcus } \\
\text { anginosus, Streptococcus intermedius, } \\
\text { Streptococcus constellatus) }\end{array}$ & May 2014 \\
\hline $\begin{array}{l}\text { Tedizolid } \\
\text { phosphate }\end{array}$ & $\begin{array}{l}\text { Cubist } \\
\text { Pharmaceuticals } \\
\text { (MA, USA) }\end{array}$ & Oxazolidinone & ABSSSI & $\begin{array}{l}\text { S. aureus (including MSSA and MRSA), } \\
\text { S. pyogenes, S. agalactiae and S. anginosus } \\
\text { group (including S. inosus, S. intermedius, } \\
\text { and S. constellatus), and E. faecalis }\end{array}$ & \\
\hline Telavancin & $\begin{array}{l}\text { Theravance } \\
\text { (CA, USA) }\end{array}$ & Lipoglycopeptide & ABSSSI & $\begin{array}{l}\text { S. aureus (including MSSA and MRSA), } \\
\text { S. pyogenes, S. agalactiae, S. anginosus } \\
\text { group (includes S. anginosus, S. intermedius, } \\
\text { and S. constellatus), E. faecalis } \\
\text { (vancomycin-susceptible strains only) }\end{array}$ & \\
\hline
\end{tabular}


Table 5. Preclinical antibiotics currently in development against Gram-negative organisms.

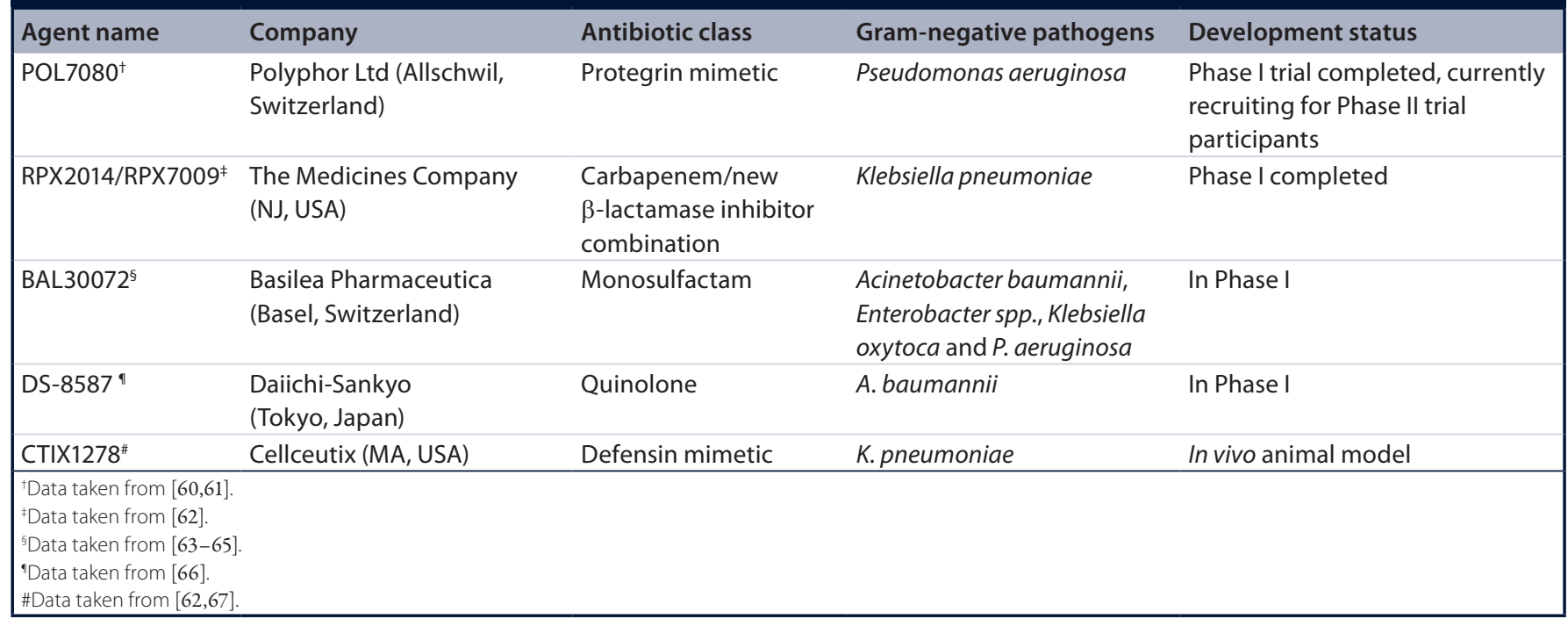

cIAI in hospitalized adults, the overall rate of clinical cure at the test-of-cure visit was $90 \%$ $(18 / 20)$ among patients with infections due to ESBL-producing Gram-negative pathogens [69].

In July 2013, the FDA designated eravacycline as a QIDP with fast-track status [70]. Patients are currently being recruited for Phase III studies comparing eravacycline with ertapenem for the treatment of cIAIs [71], and comparing eravacycline with levofloxacin for the treatment of cUTIs [72].

Plazomicin (ACHN-490) (Achaogen, CA, USA) is an aminoglycoside derivative of sisomicin, with in vitro activity against $E$. coli, $K$. pneumoniae and A. baumannii [73,74] and similar activity against ESBL-producing strains of Enterobacter spp., E. coli and K. pneumoniae [75]. Plazomicin was more active in vitro than gentamicin, tobramycin and amikacin against A. baumanni [74]. No evidence of ototoxicity or nephrotoxicity was observed in healthy subjects administered with escalating single and multiple doses of plazomicin [76].

A Phase II trial has assessed the safety and efficacy of plazomicin, compared with that of levofloxacin, in patients with cUTIs and acute pyelonephritis caused primarily by non-MDR Enterobacteriaceae [77]. Plazomicin was well-tolerated by patients and demonstrated comparable efficacy to levofloxacin, in terms of microbiological eradication of the causative pathogen and clinical outcome, specifically, resolution of baseline signs and symptoms [78]. A Phase III study is currently recruiting participants with HABP due to carbapenem-resistant Enterobacteriaceae to evaluate the efficacy and safety of plazomicin plus meropenem or tigecycline [79].

Nemonoxacin (TG-873870) (TaiGen Biotechnology Co., Ltd, Taiwan) is a novel nonfluorinated quinolone currently in clinical development for the treatment of CABP and diabetic foot infections. Nemonoxacin has broad-spectrum of activity against both Grampositive (S. aureus, S. capitis, S. pneumoniae and $E$. faecalis) and Gram-negative (E. coli) pathogens. It has been shown to have similar activity to ciprofloxacin, moxifloxacin and levofloxacin against Enterobacteriaceae [80]. A Phase III study is currently recruiting participants with CABP to evaluate the efficacy and safety of intravenous nemonoxacin with levofloxacin.

JNJ-Q2 (Furiex Pharmaceuticals, NC, USA) is another novel, broad-spectrum fluoroquinolone antibiotic that is in clinical development. It has been shown to be highly active against Gram-positive pathogens (including MRSA and MDR S. pneumoniae), Gram-negative and atypical pathogens [81]. In a Phase II clinical trial, JNJ-Q2 demonstrated efficacy and tolerability in for the treatment of ABSSSI. In 2013, JNJ-Q2 was designated a QIDP by the FDA [82].

\section{Combination antibiotics}

As well as the single-agent antibiotics, there are two $\beta$-lactam $/ \beta$-lactamase inhibitor combinations and one non- $\beta$-lactam $/ \beta$-lactamase inhibitor combination in advanced development against Gram-negative bacteria.

Ceftolozane (CXA-101, FR264205) (Cubist Pharmaceuticals, MA, USA) is a novel 
oxyimino-aminothiazolyl cephalosporin with potent in vitro activity against $P$. aeruginosa [83]. The combination of ceftolozane/tazobactam (CXA-201) showed the same in vitro potency against $P$. aeruginosa, and was also active against E. coli and $K$. pneumoniae [84]. Although the in vitro activity of ceftolozane/tazobactam was low against ESBL-producing $K$. pneumoniae, this agent retained activity against ESBL-producing E. coli [85]. Phase II trial data for ceftolozane/ tazobactam plus metronidazole, versus meropenem, demonstrated clinical cure rates of $89 \%$ (47/53) and 96\% (23/24), respectively, among patients with cIAI at the test-of-cure visit [86]. Ceftolozane/tazobactam demonstrated microbiological success against E. coli (90\% [34/38]), $K$. pneumoniae (100\% [8/8]) and $P$. aeruginosa $(100 \%$ [4/4]).

In June 2012, ceftolozane/tazobactam was designated a QIDP by the FDA [87]. In June 2014 , it was announced that the FDA had accepted a new drug application, with priority review, for ceftolozane/tazobactam for the treatment of cUTI and cIAI, based on the Phase III trial data [88].

Relebactam (Merck \& Co., Inc., NJ, USA), formerly known as MK-7655, is a novel diazabicyclooctane $\beta$-lactamase inhibitor. In combination with imipenem, MK-7655 exhibited in vitro activity against $\mathrm{AmpC}$-producing $P$. aeruginosa and KPC-producing K. pneumoniae [89]. In September 2014, the FDA designated relebactam as a QIDP [90]. Phase II clinical studies are currently evaluating relebactam, in combination with imipenem/cilastatin, for the treatment of cUTI and cIAI.

Avibactam (previously known as NXL104) is another diazabicyclooctane $\beta$-lactamase inhibitor, with a spectrum of antimicrobial activity comprising class A ESBLs, class A carbapenemases, class $\mathrm{C}$ and some class D enzymes [91]. Ceftazidime-avibactam (AstraZeneca, London, UK/Forest Laboratories, NY, USA) is a combination of an established $\beta$-lactam antibiotic with a novel non- $\beta$-lactam $\beta$-lactamase inhibitor. In vitro studies confirm that avibactam enhances the activity of ceftazidime against most $\beta$-lactamase-producing Enterobacteriaceae [92] including KPC-producing K. pneumoniae [93], and reverses AmpC-mediated resistance in $P$. aeruginosa [94].

Ceftaroline-avibactam (AstraZeneca, London, UK/Forest Laboratories), a combination of a new broad-spectrum cephalosporin ceftaroline with avibactam is also in development [95]. Phase II clinical trials comparing ceftaroline-avibactam with doripenem in adult patients with cUTI was completed in 2014; however, the results of this study are not yet available. In vitro studies confirm that ceftaroline-avibactam has potent activity against Enterobacteriaceae including those that produce $\mathrm{KPC}$, various ESBL types and AmpC [95].

Phase II trials have been conducted comparing ceftazidime-avibactam plus metronidazole, versus meropenem, for the treatment of cIAI [96], and comparing ceftazidime-avibactam for cUTI against imipenem-cilastatin [97]. Among cIAI patients administered with either ceftazidimeavibactam or meropenem, a favorable microbiological response (defined as eradication of the baseline pathogen) was observed at the test-of-cure visit in $90-100 \%$ of patients with E. coli, K. pneumoniae and P. aeruginosa (including ceftazidime-non-susceptible isolates) [96]. Rates of favorable microbiological response were $76-86 \%$ for the ceftazidime-avibactam treatment group and $70-80 \%$ for the imipenemcilastatin group among patients with cUTI due to ceftazidime-susceptible and resistant $E$. coli [97].

In 2013, ceftazidime/avibactam was designated a QIDP [98]. A Phase III study comparing the safety, efficacy and tolerability of ceftazidime/avibactam with metronidazole, compared with meropenem, has recently been completed among patients with cIAI [99]. Participants are now being recruited for Phase III clinical trials comparing ceftazidime/avibactam against doripenem among patients with cUTI [100], and against meropenem for patients with HABP, including VABP [101].

Omadacycline (Paratek Pharmaceuticals Inc, CA, USA) is a new tetracycline derivative antibiotic belonging to the aminomethycycline class currently in clinical development for the treatment of infectious diseases including ABSSSI, CABP and UTI [80]. In vitro studies reported a broad-spectrum of activity of omadacycline including against Gram-positive, Gram-negative, anaerobic and atypical pathogens. Phase II study comparing omadacycline with linezolid for the treatment of SSSI initially reported to show comparable efficacy across all the study populations, however this study has been terminated [80,102].

\section{Conclusion}

Several promising new agents are in clinical development, for example, single agent 
antibiotics such as ceftobiprole and eravacycline, and combination antibiotics such as ceftolozane/tazobactam and ceftazidime/avibactam. Historically, $\beta$-lactam/ $\beta$-lactamase inhibitor combinations, such as amoxicillin/ clavulanic acid and piperacillin/tazobactam, have proven more resilient than single agents to $\beta$-lactamase-mediated resistance. It will be interesting to see how the combinations of new cephalosporin/old $\beta$-lactamase inhibitor (e.g., ceftolozane/tazobactam) versus old cephalosporin/new non- $\beta$-lactam $\beta$-lactamase inhibitor (e.g., ceftazidime/avibactam) compete in the HAI arena. Should these new agents be approved, the burden of HAIs could be significantly reduced and patient welfare considerably improved, but the development of new agents must still progress in the meantime.

Recent data from the NHSN gave an estimate of 721,800 HAIs among inpatients of all ages in US acute care hospitals in 2011, equating to 1 in 25 patients [5]. This study used different patient populations, different definitions of HAIs and different data collection and analysis methods to the earlier study by Klevens et al. [2] which may explain, in part, the difference in the total number of estimated HAIs.

The above statistics show that HAIs are significant healthcare issues that are still unresolved due, in part, to the causative Gram-negative pathogens that have acquired antimicrobial drug resistance. Policies and recommendations have been proposed by healthcare agencies such as the AHRQ, CDC, HHS and IDSA to aid healthcare facilities to address the burden of HAIs. These policies alone are not enough, however, and there has been a resurgence in the efforts of the US government and the FDA to encourage drug developers to progress new antibiotics for combating resistant Gram-negative pathogens.

Through good infection control, blocking and tackling, and novel programs like CUSP, healthcare is making a dent in prevention. Many simple measures like hand washing remain imperative. As US healthcare moves to pay-forperformance, time will tell if this impacts real infection rates and the resultant goal of saving lives.

\section{Future perspective}

The future will entail better diagnostic testing to detect the DNA or RNA of resistant organisms at the point of care before culture results, allowing for more targeted therapy, thus reducing resistance $[103,104]$. Pharmacogenetics will usher in the day when we know a patient's genetic makeup will make them more likely to respond to certain antibiotics $[105,106]$. Host factors that affect bacterial resistance, like gut flora [107], will be explored, and interactions with our immune system. Cold plasma [108], ionization [109] and ultraviolet light [27] will kill organisms in the air, surfaces, and even topically on

\section{EXECUTIVE SUMMARY}

\section{Healthcare-associated infections}

- Healthcare-associated infections (HAls) occur in hospitalized patients within $48 \mathrm{~h}$ of admission, who have also been in contact with the healthcare service in the previous year.

- HAls caused by Gram-negative organisms are associated with antibiotic resistance.

\section{Controlling healthcare-associated infections}

- US healthcare agencies have introduced measures, such as surveillance systems, prevention programs, antimicrobial stewardship policies and quality measures to address the burden of all HAls.

- There are data showing reductions in the rates of HAls in US healthcare institutions, as a result of these implementing control and preventative measures.

\section{Antimicrobial agents for healthcare-associated infections}

- There has been a gradual decline in the development of new antibiotics approved by the US FDA.

- This decrease has been addressed by the introduction of new US government and FDA policies to aid pharmaceutical companies in the resumption of their antimicrobial research programs, particularly against Gram-negative pathogens.

- Several new antibiotics are currently in development, with both single-agent and combination inhibitors in the clinical pipeline. 
patients. Infectious burdens will be detected in those same environments at a time when hands are cleared to be clean via sensors. Finally, many novel uses of organisms' genetic sequences will lead to new antimicrobials.

\section{Disclaimer}

The authors did not receive any payment for this manuscript. The authors designed, developed and approved all the content of the manuscript.

\section{Financial \& competing interests disclosure}

AN Amin: Speaker's bureau/consulting for Forest Laboratories, LLC and Cubist Pharmaceuticals, Inc. $D$ Deruelle: Speaker's bureau for Sanofi and Cubist
Pharmaceuticals, Inc., and advisory board/consultant for AtmosAir Limited. The authors have no other relevant affiliations or financial involvement with any organization or entity with a financial interest in or financial conflict with the subject matter or materials discussed in the manuscript apart from those disclosed.

Micron Research Limited assisted in the preparation of the manuscript. This assistance was funded by Forest Laboratories, LLC.

\section{Open access}

This work is licensed under the Creative Commons Attribution-NonCommercial 3.0 Unported License. To view a copy of this license, visit http://creativecommons.org/ licenses/by-nc-nd/3.0/

\section{References}

Papers of special note have been highlighted as:

- of interest

1 Cardoso T, Almeida M, Friedman ND et al. Classification of healthcare-associated infection: a systematic review 10 years after the first proposal. BMC Med. 12, 40 (2014).

2 Klevens RM, Edwards JR, Richards CL Jr et al. Estimating health care-associated infections and deaths in U.S. hospitals, 2002. Public Health Rep. 122(2), 160-166 (2007).

- Study using data, mainly from the National Nosocomial Infections Surveillance system, to estimate the number of healthcareassociated infections in US hospitals in 2002 , in total, as well as by patient age, location of care and type of infection.

3 Sievert DM, Ricks P, Edwards JR et al. National Healthcare Safety Network Team and Participating National Healthcare Safety Network Facilities. Antimicrobial-resistant pathogens associated with healthcareassociated infections: summary of data reported to the National Healthcare Safety Network at the Centers for Disease Control and Prevention, 2009-2010. Infect. Control Hosp. Epidemiol. 34(1), 1-14 (2013).

- Study of healthcare-associated infections in US hospitals, using data reported to the National Healthcare Safety Network between 2009 and 2010, showing infection types and associated pathogens, including drug-resistant phenotypes.

4 Rice LB. Federal funding for the study of antimicrobial resistance in nosocomial pathogens: no ESKAPE. J. Infect. Dis. 197(8), 1079-1081 (2008).

5 Magill SS, Edwards JR, Bamberg W et al. Emerging Infections Program HealthcareAssociated Infections and Antimicrobial Use
Prevalence Survey Team. Multistate point-prevalence survey of health careassociated infections. N. Engl. J. Med. 370(13), 1198-1208 (2014).

- Study, conducted with the Emerging Infections Program, estimating the number of healthcare-associated infections in US acute care hospitals in 2011, listing patient demographics, types of infections and causative pathogens.

6 Boucher HW, Talbot GH, Bradley JS et al. Bad bugs, no drugs: no ESKAPE! An update from the Infectious Diseases Society of America. Clin. Infect. Dis. 48(1), 1-12 (2009).

7 Mermel LA, Allon M, Bouza E et al. Clinical practice guidelines for the diagnosis and management of intravascular catheter-related infection: 2009 update by the Infectious Diseases Society of America. Clin. Infect. Dis. 49(1), 1-45 (2009).

8 O'Grady NP, Alexander M, Burns LA et al. Guidelines for the prevention of intravascular catheter-related infections. Clin. Infect. Dis. 52(9), e162-e193 (2011).

9 American Thoracic Society; Infectious Diseases Society of America. Guidelines for the management of adults with hospitalacquired, ventilator-associated, and healthcare-associated pneumonia. Am. J. Respir. Crit. Care Med. 171(4), 388-416 (2005).

- Clinical practice guidelines, published by the American Thoracic Society and the Infectious Diseases Society of America, to guide the treatment and management of hospital-acquired, ventilator-associated and healthcare-associated pneumonia.

10 Nicolle LE. AMMI Canada Guidelines Committee. Complicated urinary tract infection in adults. Can. J. Infect. Dis. Med. Microbiol. 16(6), 349-360 (2005).

- Clinical practice guidelines to guide the treatment and management of complicated urinary tract infections.

11 Lane DR, Takhar SS. Diagnosis and management of urinary tract infection and pyelonephritis. Emerg. Med. Clin. North. Am. 29(3), 539-552 (2011).

12 Mazzulli T. Diagnosis and management of simple and complicated urinary tract infections (UTIs). Can. J. Urol. 19(Suppl. 1), 42-48 (2012).

13 Solomkin JS, Mazuski JE, Bradley JS et al. Diagnosis and management of complicated intra-abdominal infection in adults and children: guidelines by the Surgical Infection Society and the Infectious Diseases Society of America. Clin. Infect. Dis. 50(2), 133-164 (2010).

- Clinical practice guidelines, published by the Surgical Infection Society and the Infectious Diseases Society of America, to guide the treatment and management of complicated intraabdominal infections.

14 Tokars JI, Richards C, Andrus M et al. The changing face of surveillance for health care-associated infections. Clin. Infect. Dis. 39(9), 1347-1352 (2004).

15 Centers for Disease Control and Prevention. Emerging infections programs. www.cdc.gov/ncezid/dpei/eip/

16 Centers for Disease Control and Prevention. CDC's healthcare-associated infection progress report: questions and answers. www.cdc.gov/hai/surveillance/QA

17 US Department of Health \& Human Services. Operating divisions. www.hhs.gov/about/foa/opdivs/index.html 
18 March A. Stories of success: using CUSP to improve safety. www.ahrq.gov/professionals/quality-patient

19 Pronovost $\mathrm{P}$, Needham D, Berenholtz $\mathrm{S}$ et al. An intervention to decrease catheter-related bloodstream infections in the ICU. N. Engl. J. Med. 355(26), 2725-2732 (2006).

20 Goeschel CA, Pronovost PJ. Harnessing the potential of health care collaboratives: lessons from the Keystone ICU project. In: Advances in Patient Safety: New Directions and Alternative Approaches (Volume 2: Culture and Redesign). Henriksen K, Battles JB, Keyes MA, Grady ML (Eds). Agency for Healthcare Research and Quality, MD, USA (2008).

21 ON the CUSP: Stop HAI. www.onthecuspstophai.org

22 American Urological Association.

Antimicrobial prophylaxis. www.auanet.org/education/guidelines

23 Jarlier V, Trystram D, Brun-Buisson C et al. Curbing methicillin-resistant Staphylococcus aureus in 38 French hospitals through a 15 -year institutional control program. Arch. Intern. Med. 170 (6), 552-559 (2010).

24 Climo MW, Yokoe DS, Warren DK et al. Effect of daily chlorhexidine bathing on hospital-acquired infection. $N$. Engl. J. Med. 368(6), 533-542 (2013).

25 Milstone AM, Elward A, Song X et al. Daily chlorhexidine bathing to reduce bacteraemia in critically ill children: a multicenter, cluster-randomised, crossover trial. Lancet 381(9827), 1099-1106 (2013).

26 Huang SS, Septimus E, Kleinman K et al. Targeted versus universal decolonization to prevent ICU infection. N. Engl. J. Med. 368(24), 2255-2265 (2013).

27 Anderson DJ, Gergen MF, Smathers E et al. Decontamination of targeted pathogens from patient rooms using an automated ultravioletC-emitting device. Infect. Control Hosp. Epidemiol. 34(5), 466-471 (2013).

28 Centers for Disease Control and Prevention. Duke University Prevention Epicenter. www.cdc.gov/hai/epiCenters

29 Centers for Disease Control and Prevention. Healthcare-associated infections (HAIs). www.cdc.gov/HAI/prevent

30 Association of State and Territorial Health Officials (ASTHO) and Centers for Disease Control and Prevention (CDC). Eliminating healthcare-associated infections: state policy options. Association of State and Territorial Health Officials (ASTHO), VA, USA (2011). www.cdc.gov/hai/pdfs/toolkits
31 Office of Disease Prevention and Health Promotion. Healthcare-associated infections targets. www.health.gov/hai/pdfs/HAI-Targets.pdf

32 Office of Disease Prevention and Health Promotion. National action plan to prevent health care-associated infections. www.health.gov/hai/pdfs

33 Dellit TH, Owens RC, McGowan JE Jr et al. Infectious Diseases Society of America; Society for Healthcare Epidemiology of America. Infectious Diseases Society of America and the Society for Healthcare Epidemiology of America guidelines for developing an institutional program to enhance antimicrobial stewardship. Clin. Infect. Dis. 44(2), 159-177 (2007).

- Guidelines, published by the Infectious Diseases Society of America and the Society for Healthcare Epidemiology of America, to develop hospital-based programs to enhance antimicrobial stewardship.

34 Nowak MA, Nelson RE, Breidenbach JL, Thompson PA, Carson PJ. Clinical and economic outcomes of a prospective antimicrobial stewardship program. Am. J. Health Syst. Pharm. 69(17), 1500-1508 (2012).

35 Prasad N, Warren N, Kula A et al. Impact of antibiotic stewardship program interventions on multi-drug resistant organism resistance rates and hospital costs. Presented at: $54 \mathrm{th}$ Interscience Conference on Antimicrobial Agents and Chemotherapy. Washington, DC, USA, 5-9 September 2014.

36 Pasquale TR, Trienski TL, Olexia DE et al. Impact of an antimicrobial stewardship program on patients with acute bacterial skin and skin structure infections. Am. J. Health Syst. Pharm. 71(13), 1136-1139 (2014).

37 Septimus E, Weinstein RA, Perl TM, Goldman DA, Yokoe DS. Approaches for preventing healthcare-associated infections: go long or go wide? Infect. Control Hosp. Epidemiol. 35(7), 797-801 (2014).

38 Kuper KM, Septimus EJ. Health-careassociated infections: the legislative perspective and the pharmacist's role. Am. J. Health Syst. Pharm. 66(5), 488-494 (2009).

39 The Hospitalist. Medicare penalties make hospital-aquired-infection solutions a priority. www.the-hospitalist.org/details

40 Centers for Medicare \& Medicaid Services. CMS final rule to improve quality of care during hospital inpatient stays. www.cms.gov/newsroom

41 Centers for Medicare \& Medicard Services. Hospital inpatient quality reporting program. www.cms.gov/Medicare/Quality-Initiatives
42 Centers for Medicare \& Medicard Services. Hospital value-based purchasing. www.cms.gov/Medicare/Quality

43 Centers for Medicare \& Medicard Services. Readmissions reduction program. www.cms.gov/Medicare/Medicare

44 Boston Scientific. FY2015 final hospital inpatient rule summary. www.bostonscientific.com/content/dam

45 Spellberg B, Powers JH, Brass EP, Miller LG, Edwards JE Jr. Trends in antimicrobial drug development: implications for the future. Clin. Infect. Dis. 38(9), 1279-1286 (2004).

46 CenterWatch. FDA approved drugs for infections and infectious diseases. www.centerwatch.com/drug-information

47 Teflaro $^{\circledR}$ (ceftaroline fosamil) for injection. www.teflaro.com

48 Basilea Pharmaceutica. Ceftobiprole. www.basilea.com/Development/Ceftobiprole

49 BioSpace. Janssen-Cilag AG announces CHMP delays European Commission decision process to approve ZEVTERATM. www.biospace.com/News

50 Perez F, Salata RA, Bonomo RA. Current and novel antibiotics against resistant Gram-positive bacteria. Infect. Drug Resist. 1, 27-44 (2008).

51 Bartlett JG, Gilbert DN, Spellberg B. Seven ways to preserve the miracle of antibiotics. Clin. Infect. Dis. 56(10), 1445-1450 (2013).

52 Williams KJ, Bax RP. Challenges in developing new antibacterial drugs. Curr. Opin. Investig. Drugs. 10(2), 157-163 (2009).

53 Livermore DM. British Society for Antimicrobial Chemotherapy Working Party on The Urgent Need: regenerating antibacterial drug discovery and development. Discovery research: the scientific challenge of finding new antibiotics. J. Antimicrob. Chemother. 66(9), 1941-1944 (2011).

54 Slama TG. Gram-negative antibiotic resistance: there is a price to pay. Crit. Care 12(Suppl. 4), S4 (2008).

55 Sharma P, Towse A. New drugs to tackle antimicrobial resistance: analysis of EU policy options.

www.ohe.org/publications/article

56 Food and Drug Administration. News release: new FDA task force will support innovation in antibacterial drug development. www.fda.gov/NewsEvents/Newsroom

57 Food and Drug Administration. Guidance for industry: applications covered by Section 505(b)(2). www.fda.gov/downloads/Drugs/Guidances

58 Infectious Diseases Society of America. The 10 x '20 Initiative: pursuing a global 
commitment to develop 10 new antibacterial drugs by 2020. Clin. Infect. Dis. 50 (8), 1081-1083 (2010).

Boucher HW, Talbot GH, Benjamin DK Jr et al. Infectious Diseases Society of America. 10 x '20 Progress - development of new drugs active against gram-negative bacilli: an update from the Infectious Diseases Society of America. Clin. Infect. Dis. 56(12), 1685-1694 (2013).

60 Butler MS, Blaskovich MA, Cooper MA. Antibiotics in the clinical pipeline in 2013. J. Antibiot. (Tokyo) 66(10), 571-591 (2013).

- Review of recently launched antibiotics, and new compounds and inhibitor combinations in active clinical development.

61 ClinicalTrials.gov NCT02096328. http://clinicaltrials.gov/show/NCT02096328

62 Daiichi-Sankyo. Major R\&D pipeline. www.daiichisankyo.com/rd/pipeline/pdf

63 Page MG, Dantier C, Desarbre E. In vitro properties of BAL30072, a novel siderophore sulfactam with activity against multiresistant gram-negative bacilli. Antimicrob. Agents Chemother. 54(6), 2291-2302 (2010).

64 Mushtaq S, Woodford N, Hope R, Adkin R, Livermore DM. Activity of BAL30072 alone or combined with $\beta$-lactamase inhibitors or with meropenem against carbapenemresistant Enterobacteriaceae and nonfermenters. J. Antimicrob. Chemother. 68(7), 1601-1608 (2013).

65 Basilea Pharmaceutica. BAL30072. www.basilea.com/Development/BAL30072

66 Cellceutix. http://cellceutix.com

67 Higuchi S, Onodera Y, Chiba M, Hoshino K, Gotoh N. Potent in vitro antibacterial activity of DS-8587, a novel broad-spectrum quinolone, against Acinetobacter baumannii. Antimicrob. Agents Chemother. 57(4), 1978-1981 (2013).

68 Sutcliffe JA, O’Brien W, Fyfe C, Grossman TH. Antibacterial activity of eravacycline (TP-434), a novel fluorocycline, against hospital and community pathogens. Antimicrob. Agents Chemother. 57(11), 5548-5558 (2013).

69 Solomkin JS, Ramesh MK, Cesnauskas G et al. Phase 2, randomized, double-blind study of the efficacy and safety of two dose regimens of eravacycline versus ertapenem for adult community-acquired complicated intra-abdominal infections. Antimicrob. Agents Chemother. 58(4), 1847-1854 (2014).

70 Business Wire. FDA grants QIDP designation to eravacycline, tetraphase's lead antibiotic production candidate. www.businesswire.com/news
71 ClinicalTrials.gov NCT01844856. http://clinicaltrials.gov/show/NCT01844856

72 ClinicalTrials.gov NCT01978938. http://clinicaltrials.gov/ct2/show/NCT01978938

73 Landman D, Babu E, Shah N et al. Activity of a novel aminoglycoside, ACHN-490, against clinical isolates of Escherichia coli and Klebsiella pneumoniae from New York City. J. Antimicrob. Chemother. 65(10), 2123-2127 (2010).

74 Landman D, Kelly P, Bäcker M et al. Antimicrobial activity of a novel aminoglycoside, ACHN-490, against Acinetobacter baumannii and Pseudomonas aeruginosa from New York City. J. Antimicrob. Chemother. 66(2), 332-334 (2011).

75 Galani I, Souli M, Daikos GL et al. Activity of plazomicin (ACHN-490) against MDR clinical isolates of Klebsiella pneumoniae, Escherichia coli, and Enterobacter spp. from Athens, Greece. J. Chemother. 24(4), 191-194 (2012)

76 Cass RT, Brooks CD, Havrilla NA et al. Pharmacokinetics and safety of single and multiple doses of ACHN-490 injection administered intravenously in healthy subjects. Antimicrob. Agents Chemother. 55(12), 5874-5880 (2011).

77 ClinicalTrials.gov NCT01096849. http://clinicaltrials.gov/ct2/show/NCT01096849

78 Achaogen. Plazomicin. www.achaogen.com/plazomicin

79 ClinicalTrials.gov NCT01970371. www.clinicaltrials.gov/ct2/show/NCT01970371

80 Bassetti M, Merelli M, Temperoni C, Astilean A. New antibiotics for bad bugs: where are we? Ann. Clin. Microbiol. Antimicrob. 12, 22 (2013).

81 Biedenbach DJ, Turner LL, Jones RN, Farrell DJ. Activity of JNJ-Q2, a novel fluoroquinolone, tested against Neisseria gonorrhoeae, including ciprofloxacin-resistant strains. Diagn. Microbiol. Infect. Dis. 74(2), 204-206 (2012).

82 Furiex ${ }^{\circledR}$ Pharmaceuticals. JNJ-Q2. www.furiex.com/pipeline

83 Takeda S, Nakai T, Wakai Y, Ikeda F, Hatano $\mathrm{K}$. In vitro and in vivo activities of a new cephalosporin, FR264205, against Pseudomonas aeruginosa. Antimicrob. Agents Chemother. 51(3), 826-830 (2007).

84 Sader HS, Rhomberg PR, Farrell DJ, Jones RN. Antimicrobial activity of CXA-101, a novel cephalosporin tested in combination with tazobactam against Enterobacteriaceae, Pseudomonas aeruginosa, and Bacteroides fragilis strains having various resistance phenotypes. Antimicrob. Agents Chemother. 55(5), 2390-2394 (2011).
85 Farrell DJ, Flamm RK, Sader HS, Jones RN. Antimicrobial activity of ceftolozanetazobactam tested against Enterobacteriaceae and Pseudomonas aeruginosa with various resistance patterns isolated in U.S. Hospitals (2011-2012). Antimicrob. Agents Chemother. 57(12), 6305-6310 (2013).

86 Lucasti C, Hershberger E, Miller B et al. Multicenter, double-blind, randomized, phase II trial to assess the safety and efficacy of ceftolozane-tazobactam plus metronidazole compared with meropenem in adult patients with complicated intra-abdominal infections. Antimicrob. Agents Chemother. 58(9), 5350-5357 (2014).

87 Cubist Pharmaceuticals. FDA grants QIDP designation to two cubist Phase 3 antibiotic candidates.

http://investors.cubist.com/Mobile

88 Cubist Pharmaceuticals. Ceftolozanetazobactam. www.cubist.com

89 Hirsch EB, Ledesma KR, Chang KT, Schwartz MS, Motyl MR, Tam VH. In vitro activity of MK-7655, a novel $\beta$-lactamase inhibitor, in combination with imipenem against carbapenem-resistant Gram-negative bacteria. Antimicrob Agents Chemother. 56(7), 3753-3757 (2012).

90 Business Wire. Merck's investigational beta-lactamase inhibitor relebactam (MK-7655) granted qualified infectious disease product (QIDP) and fast track designations by FDA. www.businesswire.com/news

91 Stachyra T, Levasseur P, Péchereau MC et al. In vitro activity of the $\beta$-lactamase inhibitor NXL104 against KPC-2 carbapenemase and Enterobacteriaceae expressing KPC carbapenemases. J. Antimicrob. Chemother. 64(2), 326-329 (2009).

92 Zhanel GG, Lawson CD, Adam H et al. Ceftazidime-avibactam: a novel cephalosporin/ $\beta$-lactamase inhibitor combination. Drugs 73(2), 159-177 (2013).

93 Livermore DM, Mushtaq S, Warner M et al. Activities of NXL104 combinations with ceftazidime and aztreonam against carbapenemase-producing Enterobacteriaceae. Antimicrob. Agents Chemother. 55(1), 390-394 (2011).

94 Mushtaq S, Warner M, Livermore DM. In vitro activity of ceftazidime+NXL104 against Pseudomonas aeruginosa and other non-fermenters. J. Antimicrob. Chemother. 65(11), 2376-2381 (2010).

95 Castanheira M, Sader HS, Farrell DJ, Mendes RE, Jones RN. Activity of ceftarolineavibactam tested against gram-negative 
organism populations, including strains expressing one or more $\beta$-lactamases and methicillin-resistant Staphylococcus aureus carrying various staphylococcal cassette chromosome mec types. Antimicrob. Agents Chemother. 56(9), 4779-4785 (2012).

96 Lucasti C, Popescu I, Ramesh MK, Lipka J, Sable C. Comparative study of the efficacy and safety of ceftazidime/avibactam plus metronidazole versus meropenem in the treatment of complicated intra-abdominal infections in hospitalized adults: results of a randomized, double-blind, Phase II trial. J. Antimicrob. Chemother. 68(5), 1183-1192 (2013).

97 Vazquez JA, González Patzán LD, Stricklin D et al. Efficacy and safety of ceftazidimeavibactam versus imipenem-cilastatin in the treatment of complicated urinary tract infections, including acute pyelonephritis, in hospitalized adults: results of a prospective, investigator-blinded, randomized study. Curr. Med. Res. Opin. 28(12), 1921-1931 (2012).
98 Forest Laboratories, Inc. Ceftazidime/ avibactam combination receives qualified infectious disease product (QIDP) designation from FDA. http://investor.frx.com/press-release

99 ClinicalTrials.gov NCT01499290. www.clinicaltrials.gov/ct2/show/NCT01499290

100 ClinicalTrials.gov NCT01599806. www.clinicaltrials.gov/ct2/show/NCT01599806

101 ClinicalTrials.gov NCT01808092. https://clinicaltrials.gov/ct2/show/NCT01808092

102 ClinicalTrials.gov search. https://clinicaltrials.gov/ct2/results

103 Peacock S. Health care: bring microbial sequencing to hospitals. Nature 509(7502), 557-559 (2014).

104 Zumla A, Al-Tawfiq JA, Enne VI, Kidd M, Drosten C, Breuer J et al. Rapid point of care diagnostic tests for viral and bacterial respiratory tract infections: needs, advances, and future prospects. Lancet Infect. Dis. 14(11), 1123-1135 (2014).
105 Dandekar T, Dandekar G. Pharmacogenomic strategies against microbial resistance: from bright to bleak to innovative.

Pharmacogenomics 11(9), 1193-1196 (2010).

106 Baietto L, Corcione S, Pacini G, Di Perri G, D'Avolio A, De Rosa FG. A 30-years review on pharmacokinetics of antibiotics: is the right time for pharmacogenetics? Curr. Drug Metab. 15(6), 581-598 (2014).

107 Carlet J. The gut is the epicentre of antibiotic resistance. Antimicrob. Resist. Infect. Control 1(1), 39 (2012).

108 O'Connor N, Cahill O, Daniels S, Galvin S, Humphreys $\mathrm{H}$. Cold atmospheric pressure plasma and decontamination. Can it contribute to preventing hospital-acquired infections? J. Hosp. Infect. 88(2), 59-65 (2014).

109 Denham ME, Kasali A, Steinberg JP, Cowan DZ, Zimring C, Jacob JT. The role of water in the transmission of healthcare-associated infections: opportunities for intervention through the environment. HERD 7(Suppl.), 99-126 (2013). 